\title{
Stem Cells and Lung Regeneration
}

\author{
Mohammad K. El-Badrawy ${ }^{1}$, Nesrein M. Shalabi ${ }^{1}$, Mie A. Mohamed ${ }^{2}$, \\ Amany Ragab ${ }^{1}$, Heba Wagih Abdelwahab ${ }^{1}$ \\ Departments of ${ }^{1}$ Chest Medicine, ${ }^{2}$ Pathology, Mansoura University, Mansoura, Egypt
}

Background: Tissues such as the lung, liver, and pancreas that have a low steady-state cell turnover yet can respond robustly after injury to replace damaged cells. The airway epithelium is exposed to inhaled particles and pathogens that may lead to the development of a many infectious and inflammatory respiratory diseases. Lung transplantation is an accepted modality of treatment for end-stage lung diseases. Since the early $1990 \mathrm{~s}$, more than 26,000 lung transplants have been performed at centers worldwide. However, the availability of donor tissues and organs is limited, which presents a serious limitation for widespread transplantation surgery. The appearance of bioengineered lung and tracheal tissue transplants is considered a promising alternative to the classical transplantation of donor organ/tissue. Stem cells therapy arises as a new therapeutic approach, with a wide application potential.

Keywords: Lung, Stem cells or stem cells, lung regeneration, stem cell mobilization, progenitor cells

\section{Introduction}

Daar and Greenwood (2007) stated that "regenerative medicine aims at repair, replacement of cells or organs to recover the impaired function' (1). It helps the body to form new functional tissue. It includes cell therapies, tissue engineering, gene therapy and biomedical engineering techniques" (2).

So, cell therapy and tissue engineering are parts of the field of regenerative medicine, whose aim is production of safe and effective therapies. Stem cells present in almost every type of tissues and represent an endogenous system of regeneration and repair. Therefore, stem cells represent great hope for the future of translational medicine (3).

\footnotetext{
Accepted for publication March 1, 2016, Published online May 30, 2016 Correspondence to Heba Wagih Abdelwahab

Department of Chest Medicine, Mansoura University, Mansoura 35516, Egypt

Tel: +00201011930335, Fax: +002-050-2267016

E-mail: wagihheba84@gmail.com

(c) This is an open-access article distributed under the terms of the Creative Commons Attribution Non-Commercial License (http://creativecommons.org/ licenses/by-nc/4.0/), which permits unrestricted non-commercial use, distribution, and reproduction in any medium, provided the original work is properly cited.
}

\section{Lung Development and progenitor cells}

The epithelium of the whole respiratory system developed from a region of anterior ventral foregut endoderm, marked by the transcription factor Nkx2-1. After lung maturation, the epithelium differs along the proximaldistal axis, both in cellular structure and structural organization and, related to this, in stem cell composition and strategies for repair (4).

\section{Proximal-Distal pattern of the Epithelium}

The process of branching morphogenesis is the beststudied phase in lung development, by which the two primary lung buds that develop around $4 \sim 5$ weeks gestation in the human give rise to the airway tree. The buds are composed of a simple endodermal epithelium surrounded by mesoderm and a vascular plexus. These tissues are surrounded by a thin layer of mesothelium that makes a transient early contribution to mesenchymal lineages (5). All endodermal cells in lung express Nkx2-1 and this marker persists into the adult. Then, as the primary buds branch, specific patterns of gene expression appear in the endoderm of the stalks versus the buds. This proximal-distal difference is represented by the expression of Sox 2 and 
Sox9, two transcription factors required for early lung development. Sox 2 expression is confined to the proximal stalks, while Sox9 is dynamically expressed in the more highly proliferative cuboidal cells of the distal buds (6). Many other genes are differentially expressed in the tips, including Id2, which encodes a bHLH transcription factor $(4,7)$. Several studies suggested that the Id2 + tip cells are a population of multipotent progenitors. Early in development the tip cells generate daughters that translocate into the stalks and give rise to Sox $2+$ precursors of all the cell types in the intrapulmonary bronchi and bronchioles. These progeny include neuroendocrine cells, multiciliated cells, and dome-shaped secretory cells (originally called Clara cells but now known as Club cells) (4).

\section{Applying Developmental Pathways to Derive Lung Progenitors from peripheral stem cells}

Several researches have demonstrated that application of signaling factors in a way that mimics the sequence of events during early anterior foregut and lung development is important for deriving lung endoderm progenitors from PSCs. Key pathways include those downstream of Activin/ Nodal, Wnts, Bmps, and Fgfs (4, 8). PSC-derived lung endoderm can be used for basic studies of diseases such as cystic fibrosis and potentially for cell-based therapies.

\section{Progenitor Cell and Adult Lung Regeneration}

Different regions of the lung contain different populations of epithelial cells that function as adult stem cells, as defined by their ability to self-renewal and differentiation during lung injury (4).

\section{Mechanisms of Lung Regeneration}

One approach is the interactions between epithelial and mesenchymal cells in the reparative niche because crosstalk between these populations is known to be an essential process for proper development of the lung. Other signaling pathways, such as Wnt and Notch, known to play important roles in stem cell self-renewal and differentiation also play key roles in lung repair and regeneration. Wnt signaling is an essential regulator of early lung endoderm specification and development and has been implicated in regulating regenerative responses. Using Wnt reporter lines, several groups have demonstrated activation of canonical Wnt signaling in various compartments in the lung undergoing active regrowth and regeneration. In the airway epithelium, Wnt signaling is activated upon secretory cell depletion by naphthalene treatment. Activation of
Wnt signaling through loss of the critical transcription factor Gata6 leads to expansion of the putative BASC population after naphthalene injury (9).

\section{Types of Stem Cells}

In humans, stem cells can be subdivided into two main categories: intrinsic and extrinsic stem cells (10). A large variety of cell types has been used for regenerative medicine, including adult cells, resident tissue specific stem cells, bone marrow stem cells, Umbilical cord blood stem cells, embryonic stem cells and the recent breakthrough discovery of induced pluripotent stem cells from mature/ adult cells (iPS) (called reprogramming, induced a pluripotent state in a previously differentiated cell type) (11).

\section{Endogenous tissue stem cells}

are undifferentiated cells that have been identified in nearly all tissues and are believed to contribute to tissue maintenance and repair. In the lung, current evidence suggests that these cells may participate in tissue regeneration after injury and originate from within the lung itself, nesting in protected niches in the distal airways, called resident progenitor cells (alveolar, endothelial and interstitial). At least three distinct regions have been described that support populations of lung tissue stem cells: inter cartilaginous regions of tracheobronchial airways, neuroepithelial bodies (NEB) in bronchioles, and the bronchoalveolar duct junction. Progenitor cells such as toxin-resistant cells, Clara cells, basal cells, etc (10).

The airway epithelium is exposed to inhaled particles and pathogens that may lead to the development of a many infectious and inflammatory respiratory diseases such as chronic bronchitis, asthma, chronic obstructive pulmonary disease, and cystic fibrosis. These diseases are associated with changes in the architecture of the airway walls, which could vary from the epithelial structure remodeling to complete rawness of the basement membrane. To restore its functions, the airway epithelium has to rapidly repair the injuries and regenerate its structure and integrity (12).

Endothelial Progenitor Cells (EPCs) represent microvascular endothelial cells originating from circulating bone marrow-derived vascular progenitor cells and expressing specific surface antigens. They are divided into two cell subsets, namely early and late outgrowth EPCs, respectively, based on their morphology, timing of appearance in colony assays and immunophenotypic and functional profile (13).

Endothelial Progenitor Cells (EPCs) may be involved in 
vascular remodeling (muscularization of blood vessels, intemal hyperplasia and vasoconstriction) by differentiation into smooth muscle cells (14). The actual mechanism of circulating EPCs differentiation into either mature endothelial cells or smooth muscle cells are still a controversy, and many studies suggest that the source of EPCs (BM, peripheral blood or umbilical cord), the microenvironment of engraftment, growth factors and cytokines are perhaps the main regulators of the final decision on the fate of EPCs. Aging, cardiovascular risk factors and cigarette smoke exposure decrease the number of circulating EPCs (15).

Endothelial Progenitor Cells (EPCs) can also preferentially localize to areas of injured lung following systemic administration and may also have paracrine effects to decrease inflammation. As such, systemic administration of autologous EPCs in both adult and pediatric patients with primary pulmonary hypertension resulted in improved cardiopulmonary and symptomatic outcomes (16).

\section{Embryonic stem cells}

Previous studies show derivation of lung specific cells from embryonic stem cells using small airway growth medium. Then another medium developed for differentiation of ES into alveolar epithelium. Many of the genes expressed in embryonic progenitors are not expressed by endogenous lung stem cells or in adult lung (10).

\section{The Regenerative Role of Stem Cells}

Many studies have provided strong evidence that stem cells (and mainly mesenchymal stem cells, MSC) can mediate tissue repair through the modulation of the local environment, affecting the immune/inflammatory response, sustaining angiogenesis and establishing co-operative effects with the resident cells, overall resulting in a significant cytoprotective and pro-survival beneficial influence. The mechanism through which this regenerative result is achieved seems to depend more on the secretion of specific bioactive factors, rather than the direct differentiation of the transplanted stem cells in the host tissue, given the low incidence and poor efficiency of their survival and therapeutically relevant level of engraftment. This hypothesis is defined as paracrine effect and the composite set of the cytokines, chemokines and growth factors regulate many biological activities and secreted from stem cells (or shed from their membranes) is defined as secretome (17).

Some reports have demonstrated that the administration of stem cell-conditioned medium, which contains all the bioactive factors released by the cells in culture, can exert the same regenerative effect obtained with cell transplantation (17).

Microvesicles have been described as key regulators of the stem cell paracrine activity. The term of extracellular microvesicles (MVs) was first introduced to indicate nano-sized bodies released as shedding vesicles by various cell types into the extracellular environment (17).

\section{Stem cell therapy limitation}

\section{Tumor formation}

Stem cell characters like some of the features of cancer cells, such as long life span, relative apoptosis resistance and ability to replicate for extended periods of time. Therefore stem cells may be considered potential candidates for malignant transformation. Moreover, similar growth regulators and control mechanisms are involved in both cancer and stem cell maintenance (18).

\section{Immune responses}

The injected stem cells may directly induce an immune response or may have a modifying effect on the immune system. Both ESC-derived cells (19) and especially MSCs (20) have been reported to be immune-privileged and have a low immunogenic potential. Consequently allogenic administration may require reduced or even no immune suppression. However, upon differentiation these cells may become more immunogenic due to e.g. upregulation of a normal set of major histocompitability complex (MHC) molecules. Especially in case of cells that are not intended to be used for the same essential function or functions in the recipient as in the donor (non-homologous use) or when administered at non-physiological sites, immunogenicity of the cells may alter and thus remains unpredictable.

\section{Unwanted (de)differentiation}

It is unknown if dedifferentiation of stem cells can occur in vitro or in vivo. Dedifferentiation of somatic cells or redifferentiation into another cell type has been described (21).

\section{Donor and recipient clinical characteristics}

Evidently, if allogenic stem cells are used there is a risk of stem cell-tissue rejection which may be (partially) overcome by donor-patient matching, by immunological sequestration or by the use of immune suppressants, which all have their own drawbacks. Numerous other factors can be identified which may or may not contribute to a risk associated with the clinical application of a stem cell 
based medicinal product. These may be specific intrinsic characteristics of the stem cell based medicinal product or more extrinsic risk factors related to e.g. the manufacturing or type of application of the product. For example, when used in an autologous setting, the underlying disease, or medication may have an impact on the number and functionality of the stem cells, which can induce unwanted side effect of stem cell therapy. Another example may be the (unknown or unidentified) secretion of trophic factors and/or a variety of growth factors by the stem cells (22).

\section{Effects of Stem Cell Engraftment in the Lung}

Stem cells have many clinical implications in the lung. Treatment by cell therapy could be envisaged for acute disorders such as ARDS, or more chronic disorders such as emphysema and lung fibrosis. Nevertheless, the consensus is now that respiratory epithelial engraftment is a very rare event, and as such cellular repair is not thought to be realistic at present. Engraftment of stem cells can also be used for genetic therapy. In a mouse model, bone marrow stem cells were genetically altered with a viral vector to express GFP. These transplanted cells then engrafted in the recipient lung, differentiating into lung epithelium while maintaining long-term transgene expression. Stem cells may therefore act as genetic vectors in certain diseases needing replacement of a protein or DNA. Within respiratory medicine, cystic fibrosis $(\mathrm{CF})$ is a devastating illness that may similarly benefit from such an approach. As well as cellular and genetic therapy for lung diseases, an appreciation of stem cells may improve the understanding and treatment of certain malignancies. There is an increasing belief that cancers contain a small number of cancer stem cells and that the majority of cells within the tumor are not capable of tumor initiation. Furthermore, these cancer stem cells are often relatively quiescent, suggesting that conventional anti proliferative chemotherapy agents may spare these cells, leading to tumor recurrence. A greater understanding of the role of cancer stem cells may help to develop specific therapies targeting them and not the rapidly proliferating daughter cells. In this instance, the detection of cancer stem cells pecific surface markers or unique molecular targets would allow directed molecular therapy (23).

\section{Potential conflict of interest}

The authors have no conflicting financial interest.

\section{References}

1. Daar AS, Greenwood HL. A proposed definition of regenerative medicine. J Tissue Eng Regen Med 2007;1: 179-184

2. House of lords Science and Technology committee : 1st Report of Session Regenerative medicine Report. 2013.

3. Ramakrishna V, Janardhan PB, Sudarsanareddy L. Stem cells and regenerative medicine. Annual Review \& Research in Biology 2011;1:79-110

4. Hogan BL, Barkauskas CE, Chapman HA, Epstein JA, Jain R, Hsia CC, Niklason L, Calle E, Le A, Randell SH, Rock J, Snitow M, Krummel M, Stripp BR, Vu T, White ES, Whitsett JA, Morrisey EE. Repair and regeneration of the respiratory system: complexity, plasticity, and mechanisms of lung stem cell function. Cell Stem Cell 2014;15:123-138

5. Dixit R, Ai X, Fine A. Derivation of lung mesenchymal lineages from the fetal mesothelium requires hedgehog signaling for mesothelial cell entry. Development 2013;140: 4398-4406

6. Chang DR, Martinez Alanis D, Miller RK, Ji H, Akiyama $\mathrm{H}$, McCrea PD, Chen J. Lung epithelial branching program antagonizes alveolar differentiation. Proc Natl Acad Sci U S A 2013;110:18042-18051

7. Alanis DM, Chang DR, Akiyama H, Krasnow MA, Chen J. Two nested developmental waves demarcate a compartment boundary in the mouse lung. Nat Commun 2014; 5:3923

8. Ghaedi M, Calle EA, Mendez JJ, Gard AL, Balestrini J, Booth A, Bove PF, Gui L, White ES, Niklason LE. Human iPS cell-derived alveolar epithelium repopulates lung extracellular matrix. J Clin Invest 2013;123:4950-4962

9. Zhang Y, Goss AM, Cohen ED, Kadzik R, Lepore JJ, Muthukumaraswamy K, Yang J, DeMayo FJ, Whitsett JA, Parmacek MS, Morrisey EE. A Gata6-Wnt pathway required for epithelial stem cell development and airway regeneration. Nat Genet 2008;40:862-870

10. Singhal S, Achary S, Mahajan S, Wanjari A, Agarwal A. Stem cells and lung diseases. J Assoc Physicians India 2011;59:433-436

11. Lensch MW. Cellular reprogramming and pluripotency induction. Br Med Bull 2009;90:19-35

12. Chistiakov DA. Endogenous and exogenous stem cells: a role in lung repair and use in airway tissue engineering and transplantation. J Biomed Sci 2010;17:92

13. Tzouvelekis A, Ntolios P, Bouros D. Stem cell treatment for chronic lung diseases. Respiration 2013;85:179-192

14. Barberà JA, Peinado VI. Vascular progenitor cells in chronic obstructive pulmonary disease. Proc Am Thorac Soc 2011;8:528-534

15. Huertas A, Testa U, Riccioni R, Petrucci E, Riti V, Savi $\mathrm{D}$, Serra P, Bonsignore MR, Palange P. Bone marrow-derived progenitors are greatly reduced in patients with severe COPD and low-BMI. Respir Physiol Neurobiol 2010; 170:23-31

16. Weiss DJ. Concise review: current status of stem cells and 
regenerative medicine in lung biology and diseases. Stem Cells 2014;32:16-25

17. Bollini S, Gentili C, Tasso R, Cancedda R. The regenerative role of the fetal and adult stem cell secretome. J Clin Med 2013;2:302-327

18. Werbowetski-Ogilvie TE, Bossé $M$, Stewart M, Schnerch A, Ramos-Mejia V, Rouleau A, Wynder T, Smith MJ, Dingwall S, Carter T, Williams C, Harris C, Dolling J, Wynder C, Boreham D, Bhatia M. Characterization of human embryonic stem cells with features of neoplastic progression. Nat Biotechnol 2009;27:91-97

19. Mohib K, Allan D, Wang L. Human embryonic stem cell-extracts inhibit the differentiation and function of monocyte-derived dendritic cells. Stem Cell Rev 2010; 6:611-621

20. Nasef A, Ashammakhi N, Fouillard L. Immunomodulatory effect of mesenchymal stromal cells: possible mechanisms. Regen Med 2008;3:531-546

21. Pessina A, Gribaldo L. The key role of adult stem cells: therapeutic perspectives. Curr Med Res Opin 2006;22:22872300

22. Aranguren XL, Verfaillie CM, Luttun A. Emerging hurdles in stem cell therapy for peripheral vascular disease. J Mol Med (Berl) 2009;87:3-16

23. Loebinger MR, Janes SM. Stem cells for lung disease. Chest 2007;132:279-285 